\title{
Effect of Voltage Bias at MEH-PPV Layer on SPR Wavelength observed during in-situ Measurement Method in Polymer Light Emitting Diode
}

\author{
Hendro $^{1)^{*}}$, Rahmat Hidayat ${ }^{2)}$, Daniel Kurnia ${ }^{2)}$, Buchari ${ }^{3)}$, and Mitra Djamal ${ }^{1)}$ \\ 1) Theoretical High Energy Physics and Instrumentation Research Division, \\ Faculty of Mathematics and Natural Sciences, Bandung Institute of Technology, Indonesia \\ 2) Physics Magnetic and Photonic Research Division, \\ Faculty of Mathematics and Natural Sciences, Bandung Institute of Technology, Indonesia \\ 3) Analytical Chemistry Research Division \\ Faculty of Mathematics and Natural Sciences, Bandung Institute of Technology, Indonesia \\ e-mail: hendro@fi.itb.ac.id
}

Received 3 November 2010, Revised 2 December 2010, Accepted 5 January 2011

\begin{abstract}
The shifting of surface plasmon resonance (SPR) wavelength has been observed during in situ measurement in polymer light emitting diode (pLED). Examination is performed using an pLED sample which has an ITO/PEDOT:PSS/MEH-PPV/Au structure. When the voltage bias is increased from 0 to 9 volt the SPR wavelength shifts from $612 \mathrm{~nm}$ to $628 \mathrm{~nm}$ and the absorption curve shifts to lower absorbance value. From the theoretical analysis, it can be understood that the change of the SPR dip and the absorption curves correspond to the change of dielectric constant of the MEH-PPV layer. These results show that SPR wavelength depends on the metal and air dielectric constant as well as on the MEH-PPV layer. These results also imply that the SPR wavelength being evaluated can be controlled by varying the voltage bias.
\end{abstract}

Keywords: Absorption, Pled, Resonance, Wavelength shift, SPR dip

\section{Introduction}

In SPR (Surface Plasmon Resonance), evanescent wave generates Surface Plasmons (SP) wave propagating along the metal/dielectric interface. The field amplitude of the SP wave decays exponentially very quickly in the metal side but slightly slower in the dielectric side ${ }^{1-3)}$. Due to this characteristic, SPR method is widely used for characterizing very thin layer surface and metaldielectric interface, which can be found in modern sensors and analytical systems ${ }^{4,5)}$.

For practical usage for sample characterizations, a dielectric sample can be placed either on the top of the metal layer (Kretschmann configuration) or in between the prism and the metal layer (Otto configuration) ${ }^{6,7)}$. However, both configurations have the same purpose, that is, to excite the SP wave in the metal-sample interface. When the resonance takes places, the incident light will be absorbed resulting in the appearance of a spectral dip in the reflection spectrum. The SPR wavelength is very sensitive to the dielectric permittivity. Therefore, the changes in the dielectric permittivity of the sample will shift the spectral dip position, which is attributed to the shift of SPR wavelength or frequency ${ }^{8)}$. The SPR wavelength also depends on the thickness and the nanostructure of the metal layer, which is important in designing the SPR sensor ${ }^{9,10)}$. Due to its sensitivity, SPR sensor becomes a powerful tool for real time monitoring ${ }^{11,12)}$.

Although the effect of the dielectric permittivity change on the SPR wavelength shift have been widely investigated in simple configuration consisting of single layer, the same effect for the case of multilayer structure, such as in polymer light emitting diode (pLED) structure, was rarely discussed. In this paper, we report the application of SPR method to investigate the change of dielectric permittivity in the active (emissive) layer of pLED by means of insitu technique at various bias voltages. The generation of SP wave in this case is not as simple as in the case of Kretschmann configuration, because the SP wave cannot be generated directly at the active layerelectrode interface. The experimental results were analyzed by comparing the data with the theoretical calculations for explaining the shift of SPR wavelength with respect to the change of bias voltage.

\section{Theory}

In the SPR method with Kretschmann configuration, light is directed to the gold thin film via a prism with refractive index $n_{p}$ as shown in Figure 1. The reflectance $\mathrm{R}$ for 3 layers system ( $p$-prism, $m$ metal, and $a$-air) is given by ${ }^{13)}$

$$
R=\left|r_{p m a}\right|^{2}=\left|\frac{r_{p m}+r_{m a} e^{-2 i k_{m z} d}}{1+r_{p m} r_{m a} e^{-2 i k_{m z} d}}\right|^{2},
$$

with the coefficients of reflection by the interface of two adjacent mediums are

$$
r_{p m}=\frac{k_{p z} \varepsilon_{m}-k_{m z} \varepsilon_{p}}{k_{p z} \varepsilon_{m}+k_{m z} \varepsilon_{p}},
$$




$$
r_{m a}=\frac{k_{m z} \varepsilon_{a}-k_{a z} \varepsilon_{m}}{k_{m z} \varepsilon_{a}+k_{a z} \varepsilon_{m}},
$$

and

$$
\begin{aligned}
& k_{j z}=\left(\varepsilon_{j} \frac{\omega^{2}}{c^{2}}-k_{x}^{2}\right)^{1 / 2}, \\
& k_{x}=\frac{\omega}{c} n_{p} \sin \theta,
\end{aligned}
$$

where $\varepsilon_{j}$ with $j=p, m$ and $a$ is the dielectric permittivity of the prism, metal or air, respectively, and $k_{j z}$ is the wave vector component perpendicular to the interface, and $d$ is the thickness of the metal film.

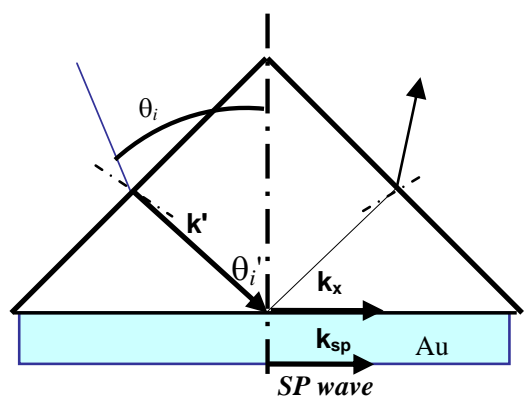

Figure 1. The generation of SP wave on the Au/air interface using Kretschmann configuration.

The SP modus can be excited by all frequencies below the plasmon frequency. The SP wave will be excited at the metal/air interface if the wave vector of the incidence light that is parallel to the interface $\left(k_{x}\right)$ is equal to the real part of the SP wave vector $\left(k_{S P}\right)$. This resonance condition occurs only at a specific frequency and incident angle $\theta_{i}^{\prime}=\theta_{S P R}$

$$
\frac{\omega}{c} n_{p} \sin \theta_{S P R}=\operatorname{Re}\left(k_{S P}\right),
$$

with

$$
k_{S P}=k_{S P}^{0}+\Delta k_{S P},
$$

where

$$
k_{S P}^{0}=\frac{\omega}{c} \sqrt{\frac{\varepsilon_{m} \varepsilon_{a}}{\varepsilon_{m}+\varepsilon_{a}}}
$$

is the resonance condition at metal/air interface and

$$
\begin{aligned}
\Delta k_{S P}= & \left(\frac{\omega}{c}\right)\left(\frac{2}{\varepsilon_{m}+\varepsilon_{a}}\right)\left(\frac{\varepsilon_{m} \varepsilon_{a}}{\varepsilon_{m}+\varepsilon_{a}}\right)^{\frac{3}{2}} \times \\
& \exp \left(i \frac{4 \pi d_{m}}{\lambda} \frac{\varepsilon_{m}}{\sqrt{\varepsilon_{m}+\varepsilon_{a}}}\right) r_{p m}\left(\mathrm{k}_{\mathrm{x}}=k_{S P}^{0}\right)
\end{aligned}
$$

is the effect of the finite thickness of the metal film $\left(d_{m}\right)$ and the presence of the prism.

\section{Experiments}

\subsection{Instrumentation}

The SPR spectroscopy system is consisted of a Tungsten Halogen light source (Ocean Optics), a polarizer (Sigma Koki), a pin-hole plate (Sigma Koki) and a motorized rotation stage (Sigma Koki) for adjusting the angles of sample and detector. A portable CCD spectrometer (USB-2000, Ocean Optics), combined with a personal computer, is used to measure the reflection spectrum.

\subsection{Measurements}

The schematic diagram for the SPR spectrum measurement on multilayer pLED is shown in Error! Reference source not found.

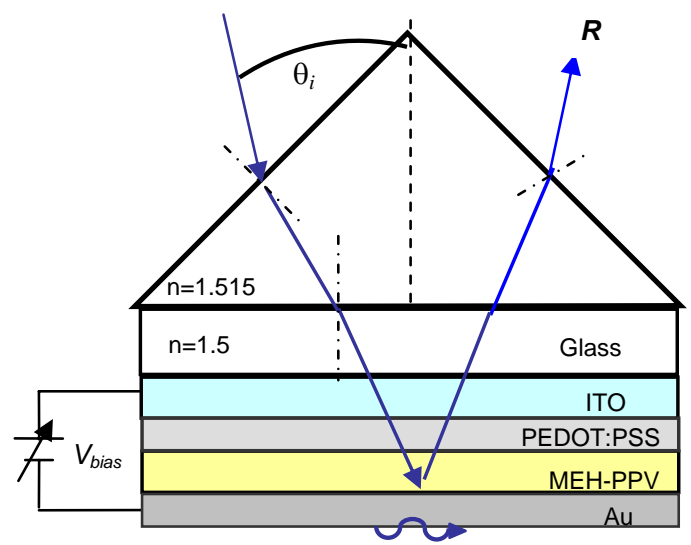

Figure 2. The schematic diagram of the SPR spectrum measurement on the pLED (ITO/PEDOT-PSS/MEH$\mathrm{PPV} / \mathrm{Au})$ structure with an applied bias voltage.

Experiment was performed by directing polychromatic light toward a BK7 prism at a fixed incident angle, namely $\theta_{i}=41^{\circ}$ (or $\theta_{i}^{\prime}=42.37^{\circ}$ ). The SPR spectrum was measured from the reflected beam passing through at various applied bias voltage in the range of $0-9 \mathrm{~V}$.

\subsection{Sample fabrication}

A glass plate with $200 \mathrm{~nm}$ thickness of indium tin oxide (ITO) layer was cut into $2 \mathrm{~cm} \times 3 \mathrm{~cm}$ size pieces. Etching process was carried out to obtain ITO line strips of $3 \mathrm{~mm} \times 30 \mathrm{~mm}$. The plate was cleaned by oxygen ( 2 minutes), UV light (10 minutes) and nitrogen (5 minutes). Poly(3,4ethylenedioxythiophene)/poly(4-styrenesulfonic acid) [PEDOT:PSS] layer was then deposited on the top of the substrate by means of spin casting with $5000 \mathrm{rpm}$ rotation speed for one minute. The sample was then heated at $100^{\circ} \mathrm{C}$ for 20 minutes. Solution of $4 \mathrm{mg}$ poly(2-methoxy-5-(2-ethylhexyl- oxy)-1,4-phenylenevinylene) [MEH-PPV] in $0.5 \mathrm{ml}$ toluene was prepared by using an ultrasonic bath for 15 minutes. This solution was then deposited on the top of the PEDOT:PSS layer also by means of spin casting with 
two steps rotation speeds, namely $500 \mathrm{rpm}$ (5 sec) and $1500 \mathrm{rpm}$ (30 sec).

Finally, gold thin layer with the thickness of only about $50 \mathrm{~nm}$ as the topmost layer was deposited by using an electron beam gun evaporation machine at $10^{-4}-10^{-5} \mathrm{~Pa}$

\section{Results and Discussions}

The measured reflectance spectrum is shown in Figure 3, which exhibit the appearance of an SPR dip at $620 \mathrm{~nm}$ for the condition of bias voltage at $0 \mathrm{~V}$. The dip position is shifted toward $628 \mathrm{~nm}$ as the bias voltage is increased gradually to $9 \mathrm{~V}$. In addition, there is also an increment in reflectance as the bias voltage increases, which means that the layer becomes more transparent at higher bias voltage.

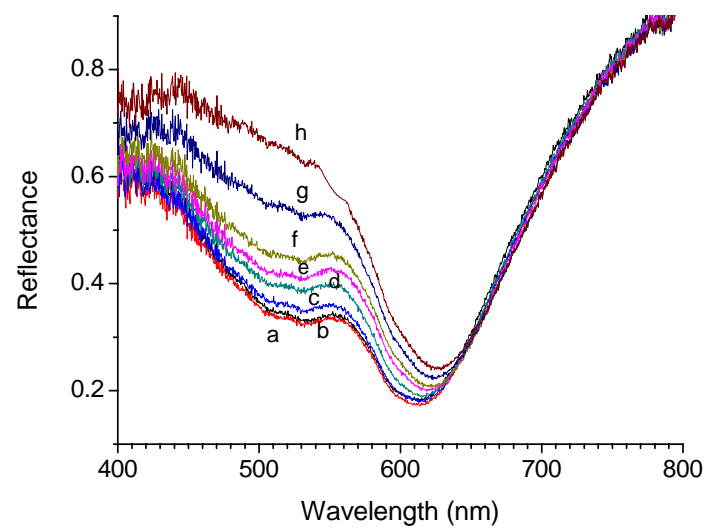

Figure 3. Reflectance spectrum measured at various bias voltages: (a) 0; (b) 1; (c) 3.5; (d) 5; (e) 6; (f) 7; (g) 8 and (h) 9V. The SPR dip position shifts from 612 $\mathrm{nm}$ to $628 \mathrm{~nm}$.

The dielectric constant and the thickness of Gold can be determined from curve fitting. ${ }^{14)}$ At the $\mathrm{Au} /$ air interface, by using the dielectric constants of $(1.515)^{2}$ for BK7 prism, $-11.74+1.67 i$ for Gold and 1 for air, the resonance was estimated to occur at incident angle toward prism/pLED interface of $43.717^{\circ}$. This result is close to the experimental data, which implies that at the incident angle of $42.37^{\circ}$, SP wave will be excited at the Au/air interface. In the case of the $\mathrm{Au} / \mathrm{MEH}-\mathrm{PPV}$ interface (with MEH-PPV refractive index $=1.8$ ), the incident angle was found to be $90^{\circ}-49.85 i$, which implies no incident angle suitable for SP excitation at Au/MEH-PPV interface.

The shift of SPR dip may indicate the change of the real part of the refractive index, which in turn implies the change in the dielectric constant of active layer inside the pLED, here the MEH-PPV emissive layer. Meanwhile, the change of dielectric constant of the electrode is neglected. due to the absence of charge accumulation there.

Application of Fresnel formulation for multilayer case (e.g. prism/dielectric/Au/air structure) turns to be unable to explain the MEH-PPV influence on the shift resonance as shown in Figure 4. The change of the real part of the subtrate refractive index does not shift the dip position, while the change of its imaginary part result in the change of reflection intensity only.

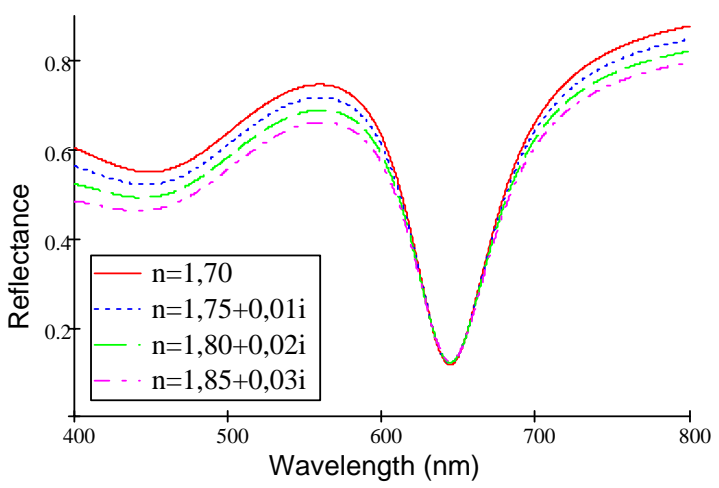

Figure 4. Reflection spectrum vs. wavelength with variation of subtrate refractive index.

Meanwhile equation (9) demonstrates that due to the thinness of the gold layer, subtrate refractive index must be taken into account, because the SP wave can reach the subtrate layer. Penetration depth of the SP wave for gold in wavelength $\lambda$ is

$$
Z_{A u}=\left|\left(\frac{2 \pi}{\lambda} \sqrt{\frac{\varepsilon_{A u}^{2}}{\varepsilon_{A u}+\varepsilon_{a i r}}}\right)\right|^{-1}
$$

For $\lambda=600 \mathrm{~nm}, \mathrm{Z}_{\mathrm{Au}}=26.5 \mathrm{~nm}$ which result in effective penetration depth $133 \mathrm{~nm}$. Because the thickness of the gold layer is $50 \mathrm{~nm}$, the SP wave will reach the MEH-PPV layer around $100 \mathrm{~nm}$. Penetration toward PEDOT:PSS layer is negligible due to the fact that very low SP energy remains after passing the MEH-PPV layer. The variation of PEDOT:PSS refractive index will not affect the SP wave vector considerably. Therefore the change of refractive index of the MEH-PPV of the pLED will shift its dip of the SPR spectrum.

If the equation (9) is applied for the multilayer pLED case, the equation of $\Delta k_{S P}$ shows its dependence to the MEH-PPV layer,

$$
\begin{aligned}
\Delta k_{S P}= & \left(\frac{\omega}{c}\right)\left(\frac{2}{\varepsilon_{A u}+\varepsilon_{a}}\right)\left(\frac{\varepsilon_{A u} \varepsilon_{a}}{\varepsilon_{A u}+\varepsilon_{a}}\right)^{\frac{3}{2}} x \\
& \exp \left(i \frac{4 \pi d_{A u}}{\lambda} \frac{\varepsilon_{A u}}{\sqrt{\varepsilon_{A u}+\varepsilon_{a}}}\right) r_{P P V-A u}
\end{aligned}
$$

with the coefficient of reflection at $\mathrm{MEH}-\mathrm{PPV} / \mathrm{Au}$ interface is

$$
r_{P P V-A u}=\frac{k_{P P V, z} \varepsilon_{A u}-k_{A u, z} \varepsilon_{P P V}}{k_{P P V, z} \varepsilon_{m}+k_{A u, z} \varepsilon_{P P V}}
$$

where $\varepsilon_{\mathrm{PPV}}$ is the dielectric constant of MEH-PPV.

The refractive index of MEH-PPV and the dielectric constant of gold are dependent of the light wavelength. For an example, the real parts of MEH- 
PPV refractive index for the $612 \mathrm{~nm}, 620 \mathrm{~nm}$ and 628 $\mathrm{nm}$ are $1.8,1.78$ and 1.76 respectively, while their imaginary parts relatively equal to zero ${ }^{13)}$. The gold dielectric constants for those wavelengths are $11.2+1.41 \mathrm{i},-12+1.39 \mathrm{i}$, dan $-12.8+1.36 \mathrm{i}^{14)}$. From the optical parameters of MEH-PPV and gold, the $\mathrm{k}_{\mathrm{SP}}$ value can be determined, while from the experimental data, the value of $k_{x}$ can be calculated as in Table 1 . Ideally, the $\mathrm{k}_{\mathrm{SP}}$ values are equal to $\mathrm{k}_{\mathrm{x}}$ in accordance to the dispersion relation shown in Figure 5. But the result in Table 1 shows less than $0.5 \%$ differences between $\mathrm{k}_{\mathrm{SP}}$ and the experimental result $\mathrm{k}_{\mathrm{x}}$.

Table 1. Comparison of $k_{S P}$ and $k_{x}$.

\begin{tabular}{|c|l|l|l|l|}
\hline $\begin{array}{c}\lambda \\
(\mathrm{nm})\end{array}$ & $\begin{array}{c}\mathrm{n} \\
\text { MEH_PPV }\end{array}$ & $\varepsilon_{\mathrm{Au}}$ & $\begin{array}{c}\mathrm{k}_{\mathrm{SP}} \times 10^{7} \\
\left(\mathrm{~m}^{-1}\right)\end{array}$ & $\begin{array}{c}\mathrm{k}_{\mathrm{x}} \times 10^{7} \\
\left(\mathrm{~m}^{-1}\right)\end{array}$ \\
\hline 612 & 1.80 & $-11.2+1.41 \mathrm{i}$ & 1.060 & 1.055 \\
\hline 620 & 1.78 & $-12.0+1.39 \mathrm{i}$ & 1.035 & 1.035 \\
\hline 628 & 1.76 & $-12.8+1.36 \mathrm{i}$ & 1.018 & 1.022 \\
\hline
\end{tabular}

The c1, c2 and c3 lines in Figure 5 represent the dispersion relation of SP for pLED system at $\mathrm{Au} /$ Air interface for different refractive index of MEH-PPV, while c4 line is the light line in the prism. The intersection of c4 line with c1, c2 and c3 indicates the shift of resonance value of the incoming wave and the SP. When the refractive index of MEH-PPV decreases, the SP wave vector or the SP resonance frequency decreases accordingly, indicating the increase of the resonance wavelength. Each intersection represents the condition for the resonance to occur.

As an example, from Table 1 , it can be seen that for $612 \mathrm{~nm}, \operatorname{Re}\left(\mathrm{k}_{\mathrm{SP} 1}\right)=1.060 \times 10^{7} \mathrm{~m}^{-1}$ is the resonance point for $\mathrm{n}_{\mathrm{MEH}-\mathrm{PPV}}=1.8$ and $\varepsilon_{\mathrm{Au}}=-11.2+1.41 \mathrm{i}$. At $620 \mathrm{~nm}$, the refractive index of MEH-PPV decreases down to 1.78 results in the shift of resonance to $\operatorname{Re}\left(\mathrm{k}_{\mathrm{SP} 2}\right)=1.035 \times 10^{7} \mathrm{~m}^{-1}$. Similarly, at 628 $\mathrm{nm}, \operatorname{Re}\left(\mathrm{k}_{\mathrm{SP} 3}\right)=1.018 \times 10^{7} \mathrm{~m}^{-1}$ is obtained. The decrease in MEH-PPV refractive index changes, result in smaller changes on $\mathrm{k}_{\mathrm{SP}}$ values, so that the SPR wavelength is shifted to higher value. The dip shift is related to the real part of MEH-PPV refractive index.

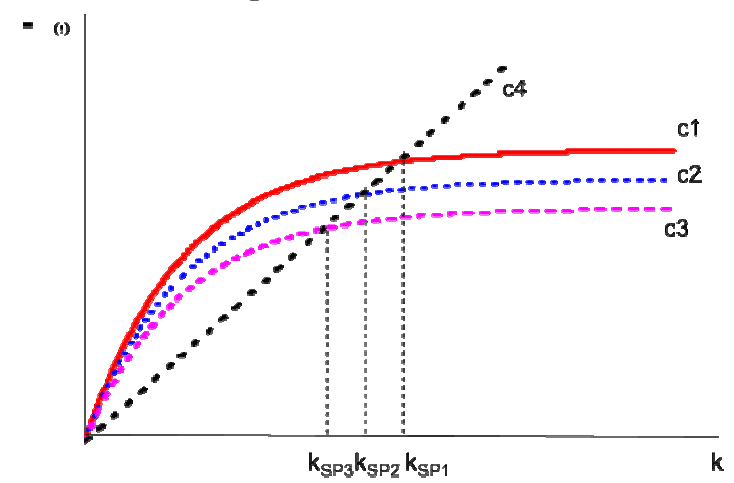

Figure 5. Dispersion relation of SP for pLED system at $\mathrm{Au}$ /Air interface for different refractive index of MEH-PPV (c1, c2 and c3), and light line in the prism (c4).
Absorption of light by a pLED can be observed from its absorption spectrum, Figure 6. When the bias voltage is increased, absorption spectrum at the 400$650 \mathrm{~nm}$ range is shifted downward, indicating the decrease in absorption. At around $510 \mathrm{~nm}$, huge absorption occur due to the MEH-PPV polymer absorption, while at above $650 \mathrm{~nm}$, practically no change of absorption observed. These changes are caused by optical characteristic changes of the MEHPPV layer when bias voltage is applied to a pLED. The imaginary part of MEH-PPV refractive index is responsible for those changes.

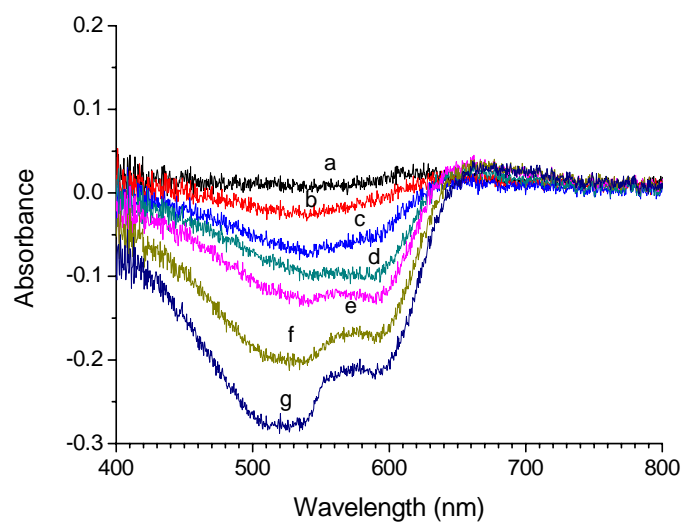

Figure 6. pLED absorption spectrum vs wavelength for various bias voltages (a) 1; (b) 3.5; (c) 5; (d) 6; (e) 7; (f) 8 dan (g) 9V.

The method described above can be used to characterize the optical properties of MEH-PPV as well as to investigate its electronic properties, due to the relation between the dielectric constant and the conductivity. Taking into account that the SP wave is excited closed to MEH-PPV/metal interface, the change of conductivity can be attributed to the change of transport properties that take place in this area.

\section{Conclusion}

The analysis of experimental data shows the shift of resonance condition of SP wave from $612 \mathrm{~nm}$ to $628 \mathrm{~nm}$ while the bias voltage applied to the pLED is increased from 0 to 9 volts. The shift is originated from the change of the real part of MEH-PPV refractive index, while the change of the absorption spectrum is caused by the change of its imaginary part. The change of MEH-PPV refractive index results in the shift of SP dispersion curve, and the resonance condition of SP wave. Analytical consideration indicates that SPR wavelength depends on the metal and the air dielectric constant as well as on the MEHPPV layer. These results also imply that the SPR wavelength being evaluated can be controlled by varying the voltage bias. The changes of reflectance and absorbance values against the bias voltage indicate the influence of the bias voltage upon the dielectric constant of MEH-PPV. 


\section{Acknowledgment}

This research is funded by Institut Teknologi Bandung through PROGRAM RISET KK ITB 2010 project No. 243/K01.7/PL/2010 and APS 2010.

\section{References}

1. H. Raether, Surface Plasmons on Smooth and Rough Surfaces and on Gratings, Springer Verlag, Berlin, 6-7, 1986.

2. R.B.M. Schasfoort and A.J. Tudos, Handbook of Surface Plasmon Resonance, The Royal Society of Chemistry, 18-19, 2008.

3. S.A. Maier, Plasmonics: Fundamentals and Applications, Springer Science Business Media LLC., 21-26, 2007.

4. G.B. Vladimiro and H.G. Rubahn, Optics and Spectroscopy at Surfaces and Interfaces, WILEYVCH Verlag GmbH \& Co. KGaA, Weinheim, 128-129, 2005.

5. O.S Wolfbeis, Surface Plasmon Resonance Based Sensors, Springer-Verlag, Berlin Heidelberg, 4548, 2006.

6. A. Otto, Excitation of Non Radiative Surface Plasma Waves in Silver by the Method Frustrated Total Reflection, Z. Phys., 216, 398-410, 1968

7. E. Kretschmann and H. Raether, Z. Naturforsch, A 23, 2135, 1968.
8. S. Ekgasit et al., Resonance Shifts in SPR Curves of Nonabsorbing, Weakly Absorbing, and Strongly Absorbing Dielectrics, Sensor and Acttuators, B 105, 532-541, 2005.

9. S. Zhu et al., Tunable Surface Plasmon Resonance of Gold Nanoparticles Self-Assembled on Fused Silica Substrate, Electrochem. Solid St., 13, 96-99, 2010.

10. E. Fontana, Thickness Optimization of Metal Films for the Development of Surface-PlasmonBased Sensors for Nonabsorbing Media, Appl. Optics, 45, 7632-7642, 2006.

11. N.S. Eum et al., Variable Wavelength Surface Plasmon Resonance (SPR) in Biosensing, BioSystems, 98, 51-55, 2009.

12. P.S. Petrou et al., Real-Time Label-Free Detection of Complement Activation Products in Human Serum by White Light Reflectance Spectroscopy, Biosensors Bioelectron., 24, 33593364, 2009.

13. P. Yeh, Optical Waves In Layered Media, John Wiley \& Son: New York, 1988, 86-87.

14. Hendro et al., Determination of the Dielectric Constant and the Thickness of Gold Film by SPR Technique, AIP Conference Proceedings, 1325, 285-288, 2010. 